\title{
Time-resolved surface-enhanced resonance Raman spectro-electrochemistry of heme proteins
}

\author{
Marc Grosserueschkamp ${ }^{\mathrm{a}, \mathrm{b}}$, Christoph Nowak ${ }^{\mathrm{a}, \mathrm{b}}$, Wolfgang Knoll ${ }^{\mathrm{b}}$ and \\ Renate L.C. Naumann ${ }^{\mathrm{a}, *}$ \\ ${ }^{a}$ Max Planck Institute for Polymer Research, Mainz, Germany \\ ${ }^{\mathrm{b}}$ Austrian Research Centers GmbH, Tech Gate Vienna, Vienna, Austria
}

\begin{abstract}
Heme proteins such as cytochrome c (cc) play a fundamental role in many biological processes. Surface-enhanced resonance Raman spectroscopy (SERRS) combined with electrochemical methods is an ideal tool to study the redox processes of heme proteins. In this context we designed a new measuring cell allowing for simultaneous electrochemical manipulation and high sensitive SERRS measurements of heme proteins. The measuring cell is based on an inverted rotating disc electrode for excitation by using a confocal Raman microscope. Furthermore, we developed a SER(R)S-active silver modified silver substrate for spectro-electrochemical applications. For this purpose silver nanoparticles (AgNPs) were adsorbed on top of a planar silver surface. The substrate was optimized for an excitation wavelength of $413 \mathrm{~nm}$ corresponding to the resonance frequency of heme structures. An enhancement factor of $10^{5}$ was achieved. The high performance of the new measuring cell in combination with the new silver substrate was demonstrated using cc as a reference system.
\end{abstract}

Keywords: Time-resolved surface-enhanced resonance Raman spectroscopy, localized surface plasmons, silver, nanoparticles, electron transfer

\section{Introduction}

Electron transfer kinetics of heme proteins adsorbed on metal surfaces can be investigated by spectroelectrochemistry [3,7]. A combination of surface-enhanced Raman scattering (SERS) and resonance Raman scattering (RRS) is particularly advantageous in this context. Surface-enhanced resonance Raman spectroscopy (SERRS) is designed to reflect vibrations of the porphyrine ring [4]. Hence the redox transitions of heme proteins triggered e.g. by electrochemical manipulation can be monitored by SERRS. The resonance frequency is an inherent property of the heme structure and is known to be in the range of $410-420 \mathrm{~nm}$. In contrast, the surface enhancement effect is dominated by the chemical composition and in particular the morphology of the SER(R)S substrate. Different mechanisms contribute to the enhanced Raman scattering of molecules adsorbed on rough metal substrates. The electro magnetic enhancement effect (EMEE) and the chemical enhancement effect (CEE), also known as charge transfer effect, are of great interest in this context $[1,10]$. The EMEE is based on collective electron oscillations in resonance with the exciting laser wavelength yielding high electro magnetic field enhancement in the proximity

\footnotetext{
${ }^{*}$ Corresponding author: Renate L.C. Naumann, Max Planck Institute for Polymer Research, Ackermannweg 10, 55128 Mainz, Germany. Tel.: +49 6131379 157; Fax:+49 6131379 100; E-mail: naumannr@ mpip-mainz.mpg.de.
} 
of the metal surface. In the literature such electron oscillations are often referred to as localized surface plasmons (LSP), particularly if they are excited within nanoscopic metal structures such as colloids or rough surface bumps. The surface enhancement effect as well as the resonance Raman scattering help to overcome the problems associated with the weak interaction cross-section of the Raman effect. However, photo-degradation of the protein by the incident laser beam is a serious problem in Raman scattering [12]. In order to overcome this problem a number of setups were designed to reduce the exposure time of the active area to the laser beam. Rotating disc electrodes (RDE) proved to be a promising approach. We designed a new Raman measuring cell using this concept [7]. Furthermore a SER(R)Sactive silver modified silver substrate was developed and optimized for the excitation with the $413 \mathrm{~nm}$ line of a $\mathrm{Kr}^{+}$laser, offering ideal properties for studying heme proteins [8].

\section{2. $\operatorname{SER}(R) S$ setup}

The setup used for Raman experiments was described in detail in a previous publication [7]. All Raman experiments were performed using the $413 \mathrm{~nm}$ line of a $\mathrm{Kr}^{+}$laser (Innova 90C, Coherent). After passing a pre-monochromator (LaserspecIII, Spectrolab Research Laboratory, Newbury, England) the laser beam was coupled into a confocal Raman microscope (LabRam, HR800, HORIBA, Jobin Yvon) equipped with a water immersion objective (Olympus LUMPLFL, $100 \mathrm{XW}, W D=1.5, N A=1$, BFOBJ). By this means the laser beam was focused on top of the modified plane of the silver rod immersed in PBS. The scattered light was filtered by a holographic notch filter and guided to an 1800 grooves $/ \mathrm{mm}$ grating providing spectral resolution. The spectrum was imaged onto a liquid nitrogen cooled back-illuminated CCD detector (Symphony, Jobin Yvon).

\section{Preparation of SER(R)S-active silver substrate}

Silver nanoparticles (AgNPs) were synthesized according to a modified version of the method of Turkevich [5] and Frens [6], who introduced citric acid as a reducing agent. $8.25 \mathrm{ml} \mathrm{37 \%} \mathrm{formaldehyde}$ was added to $250 \mathrm{ml}$ of an aqueous $\mathrm{AgNO}_{3}$ solution $(10 \mathrm{mM} / \mathrm{l})$. The mixture was heated during vigorous stirring to $100^{\circ} \mathrm{C}$ for at least $15 \mathrm{~min}$. Then $4 \mathrm{ml}$ of an aqueous solution of citric acid $(33 \mathrm{mM} / \mathrm{l})$ was added. The temperature was kept constant at $100^{\circ} \mathrm{C}$ until the color changed during the first few minutes to a bright yellow, eventually changing into a darker greenish yellow, indicating a suspension of AgNPs [8]. The NP suspension was kept at $4^{\circ} \mathrm{C}$ until used.

The top planes of $12 \mathrm{~mm}$ thick silver rods were polished as described before [7] down to a RMS roughness of 3-5 $\mathrm{nm}$. Self assembled monolayers (SAMs) were formed by immersion of the silver rods into an aqueous solution of cysteamine (CA) $(10 \mathrm{mM} / \mathrm{l})$ for $1 \mathrm{~h}$. After thorough rinsing with water the modified silver rods were immersed into the nanoparticle suspension.

\section{Adsorption of cc}

The modified silver electrodes were immersed into a solution of mercaptoethanol (ME) $(10 \mathrm{mM} / \mathrm{l})$ for at least $1 \mathrm{~h}$ to form a SAM of ME, thus displacing residual CA molecules from the surface. After extensively rinsing with water $\mathrm{cc}$ was adsorbed on the SAM of ME by immersing the functionalized silver electrode into PBS buffer $\left(20 \mathrm{mM} \mathrm{K}_{2} \mathrm{HPO}_{4} / 100 \mathrm{mM} \mathrm{NaClO}_{4} / \mathrm{pH}=7\right)$ containing $50 \mu \mathrm{M}$ cc for at least $30 \mathrm{~min}$. 


\section{The SERRS measuring cell}

The custom-made measuring cell for spectro-electrochemical applications was based on a rotating disc electrode (RDE) in the reverse orientation (Fig. 1). This assembly allowed an excitation of the sample from the top by means of the confocal Raman microscope. Photo-degradation was prevented by rotating the RDE at a constant speed of $800 \mathrm{rpm}$ by a DC-motor (FAULHABER 3863H012C 38A 1:5). A conductive bolt was inserted into the rotating axis insulated by an epoxy casting resin (3430 A\&B Hysol, Loctite). The silver modified silver electrode was mounted on top of the conductive bolt by a thread and the lower end was connected to the agile part of a mercury contact (custom-made, provided by Eco Chemie, B.V., Utrecht, The Netherlands). The cell was sealed from ambient air by a lid made from Teflon through which the immersion objective extended into the fluid reservoir of the measuring cell. Furthermore, a platinum wire used as a counter electrode, a reference electrode $(\mathrm{Ag} / \mathrm{AgCl}, \mathrm{KCl}$

i)
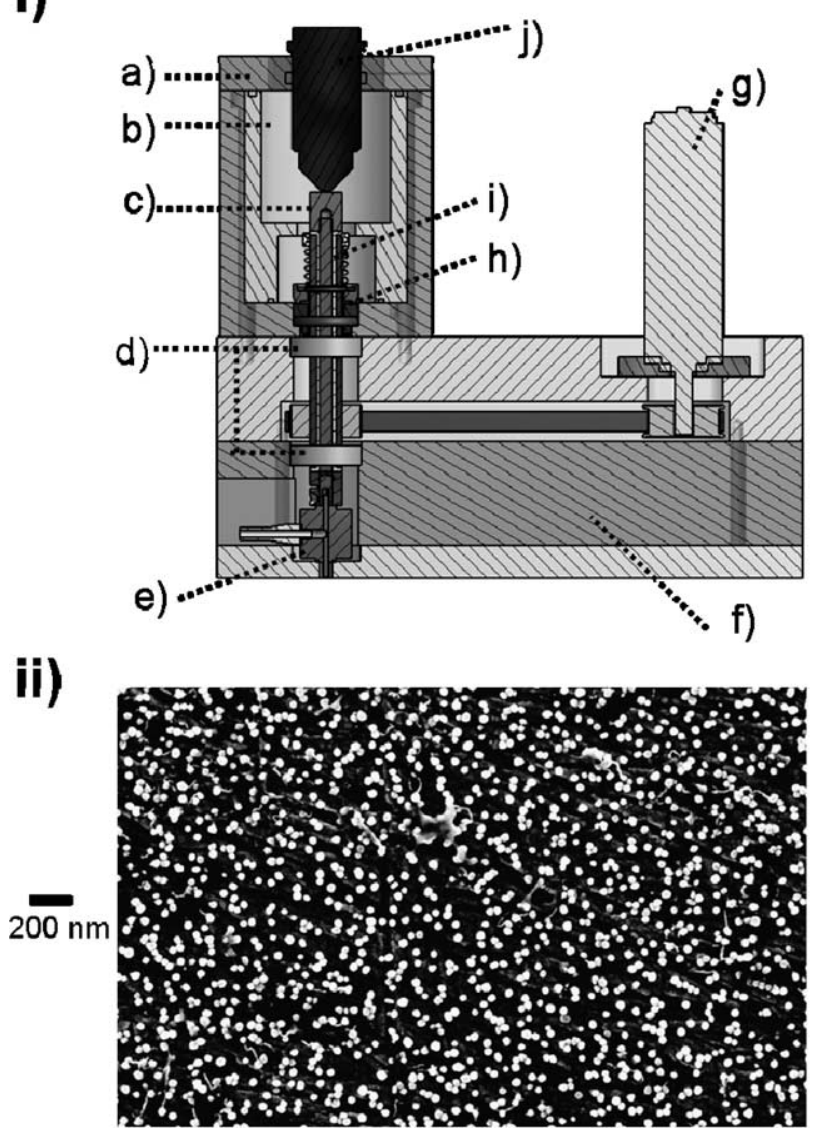

ii)

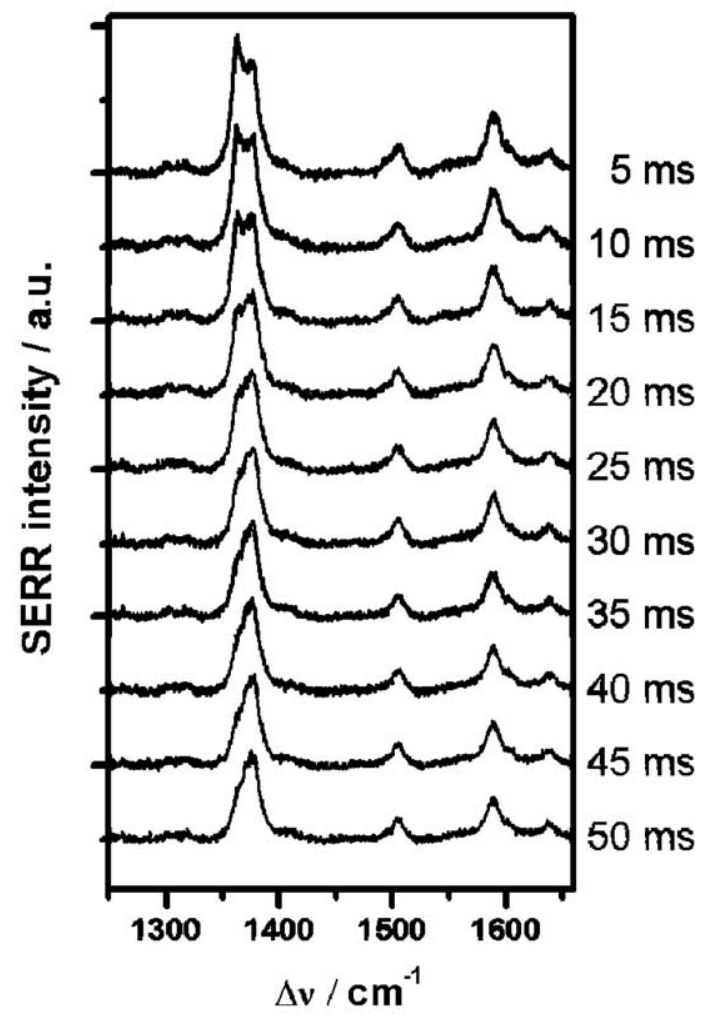

Fig. 1. (i) Cross section of the SER(R)S measuring cell. (a) Lid; (b) fluid reservoir; (c) Ag-electrode; (d) ball bearing; (e) mercury contact; (f) cell body; (g) dc motor; (h) axial face sealing; (i) drive shaft with conducting bolt; (j) immersion objective. (ii) SEM image of Ag-NP adsorbed on a SAM of CA. The immersion time in the NP suspension was $2 \mathrm{~h}$. In the SEM image the planar silver underlayer appears in black, while the AgNPs emerge as white dots. (iii) Time-resolved SERR spectra of cc. The spectra were recorded after different delay times after a potential jump from $-310 \mathrm{mV}$ to $+180 \mathrm{mV}$ (vs. $\mathrm{Ag} / \mathrm{AgCl}$ ). $\mathrm{In}$ this case the rate constant for the electron transfer process was determined to be $(46 \pm 7) \mathrm{s}^{-1}$. 
sat.) and inlet and outlet tubes were also inserted into the lid. For a more detailed description of the cell design please see reference [7].

\section{Results}

The NP suspension prepared according to the procedure described above was probed by uv-vis spectroscopy and showed a distinct extinction maximum at $\sim 410 \mathrm{~nm}$, which corresponds to the LSP resonance of the AgNPs. Hence LSPs can be excited by using the laser wavelength of $413 \mathrm{~nm}$ used for our Raman experiments, leading to an enhanced Raman signal.

Controlling the NP density per unit area by varying the immersion time of the functionalized silver electrode in the NP suspension revealed that the highest enhancement was not obtained when the NP density reached a maximum. For immersion times in the range of minutes $(<16 \mathrm{~min})$ the Raman signal showed a steady increase with the NP density. In contrast, for immersion times longer then $2 \mathrm{~h}$ higher NP densities were accompanied by a decreasing Raman signal. A detailed analysis of the NP distribution by scanning electron microscopy (SEM) showed that a large fraction of NPs formed clusters. NPs arranged in clusters feature a direct electrical contact to each other in the plane of electron oscillation. Such NP clusters can lead to a redshift of the LSP resonance frequency as reported in theoretical as well as experimental publications by other groups [2,9,11]. Uv-vis spectroscopy was used to confirm this finding. For $\mathrm{NP}$ adsorption times of $1 \mathrm{~h}$ or longer uv-vis spectra of silver substrates exhibited 2 extinction maxima at $\sim 390$ and $600 \mathrm{~nm}$. The maxima were assigned to the LSP oscillation of individual and clustered NPs, respectively. Hence NPs forming clusters did not contribute to the surface-enhanced Raman scattering. The highest number of NPs strictly separated from their next neighbors was achieved by immersion times of $\sim 2 \mathrm{~h}$ corresponding to the highest enhancement observed (Fig. 1). A detailed analysis revealed a surface enhancement factor of the new surface architecture of $\sim 10^{5}$. The enhancement factor exceeded that of silver electrodes roughened by an oxidation reduction cycle, while the electrochemical performance was nearly the same.

In combination with the newly developed SER(R)S measuring cell the optimized SER(R)S-active substrate was used to study electron transfer kinetics of cc by time-resolved SERRS. Cc adsorbed on the silver substrate was electrochemically reduced and oxidized by application of reductive and oxidative potential, respectively. The transition from the oxidized to the reduced species of cc was observed by a distinct shift in the $\nu_{4}$ band of the inherent heme structure from 1374 to $1361 \mathrm{~cm}^{-1}$. Shuttling the protein between the reduced and the oxidized form and exciting the protein sample by synchronized laser pulses of $5 \mathrm{~ms}$ each, time-resolved redox transitions were observed by SERRS (Fig. 1). We found that the orientation of the protein with the heme-site directed away from the electrode hampers the electron transfer from the silver electrode to the redox center of the protein and vice versa.

\section{References}

[1] M.G. Albrecht and J.A. Creighton, Anomalously intense Raman-spectra of pyridine at a silver electrode, Journal of the American Chemical Society 99 (1977), 5215-5217.

[2] T. Atay, J.H. Song and A.V. Nurmikko, Strongly interacting plasmon nanoparticle pairs: From dipole-dipole interaction to conductively coupled regime, Nano Letters 4 (2004), 1627-1631.

[3] A. Bonifacio, D. Millo, C. Gooijer et al., Linearly moving low-volume spectroelectrochemical cell for microliter-scale surface-enhanced resonance Raman spectroscopy of heme proteins, Analytical Chemistry 76 (2004), 1529-1531.

[4] H. Brunner, H. Sussner and A. Mayer, Resonance Raman scattering on heme group of oxyhemoglobin deoxyhemoglobin, Journal of Molecular Biology 70 (1972), 153-156. 
[5] B.V. Enustun and J. Turkevich, Coagulation of colloidal gold, Journal of the American Chemical Society 85 (1963), $3317-3328$

[6] G. Frens, Particle-size and sol stability in metal colloids, Kolloid-Zeitschrift and Zeitschrift fur Polymere 250 (1972), 736-741.

[7] M. Grosserueschkamp, M.G. Friedrich, M. Plum et al., Electron transfer kinetics of cytochrome c probed by time-resolved surface-enhanced resonance Raman spectroscopy, Journal of Physical Chemistry Ser. B 113 (2009), 2492-2497.

[8] M. Grosserueschkamp, C. Nowak, D. Schach et al., Silver surfaces with optimized surface enhancement by self-assembly of silver nanoparticles for spectroelectrochemical applications, Journal of Physical Chemistry Ser. C 113 (2009), 1769817704.

[9] L. Gunnarsson, T. Rindzevicius, J. Prikulis et al., Confined plasmons in nanofabricated single silver particle pairs: Experimental observations of strong interparticle interactions, Journal of Physical Chemistry Ser. B 109 (2005), 1079-1087.

[10] D.L. Jeanmaire and R.P. Van Duyne, Surface Raman spectroelectrochemistry: Part I. Heterocyclic, aromatic, and aliphatic amines adsorbed on the anodized silver electrode, Journal of Electroanalytical Chemistry 84 (1977), 1-20.

[11] H. Tamaru, H. Kuwata, H.T. Miyazaki et al., Resonant light scattering from individual Ag nanoparticles and particle pairs, Applied Physics Letters 80 (2002), 1826-1828.

[12] H. Wackerbarth, U. Klar, W. Gunther et al., Novel time-resolved surface-enhanced (resonance) Raman spectroscopic technique for studying the dynamics of interfacial processes: Application to the electron transfer reaction of cytochrome c at a silver electrode, Applied Spectroscopy 53 (1999), 283-291. 


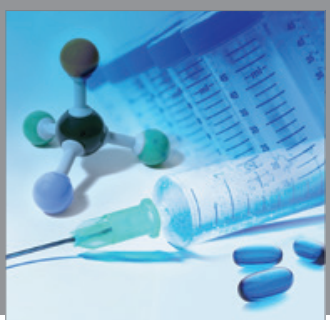

International Journal of

Medicinal Chemistry

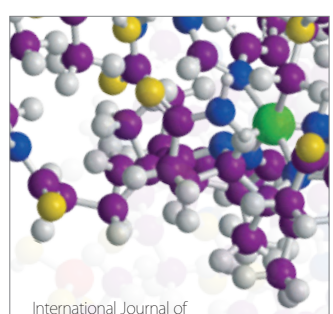

Carbohydrate Chemistry

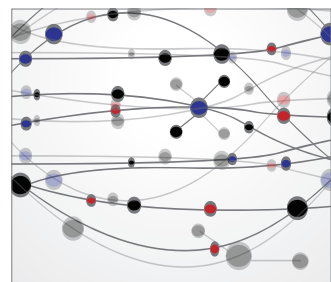

The Scientific World Journal
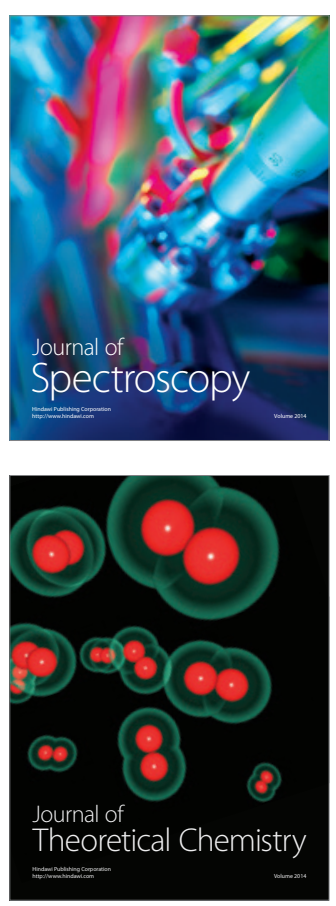
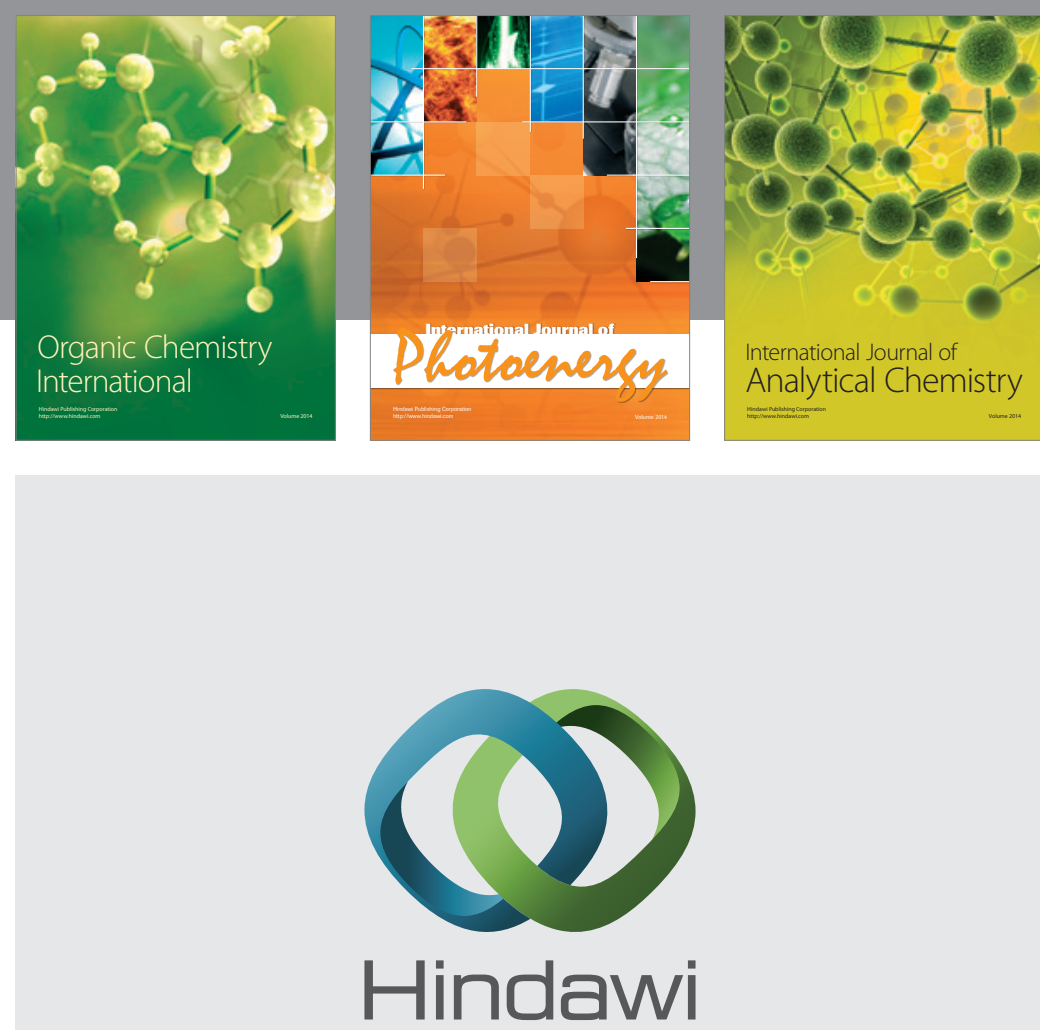

Submit your manuscripts at

http://www.hindawi.com
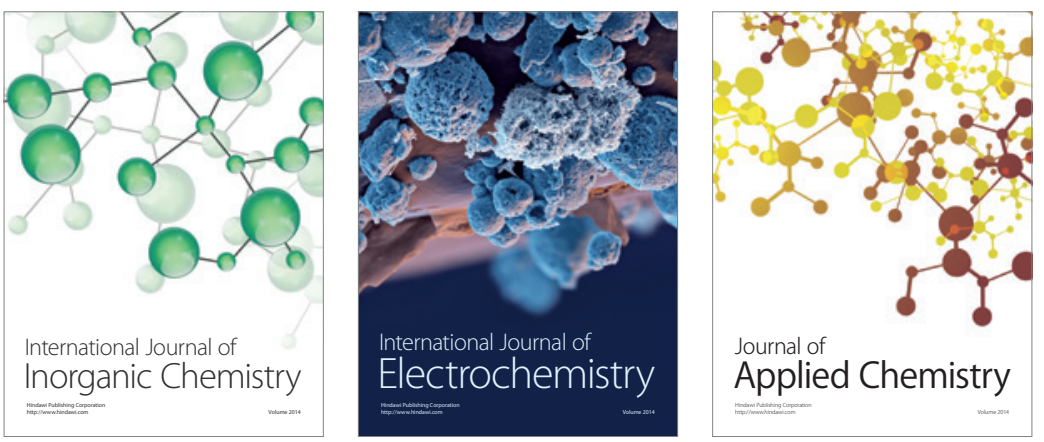

Journal of

Applied Chemistry
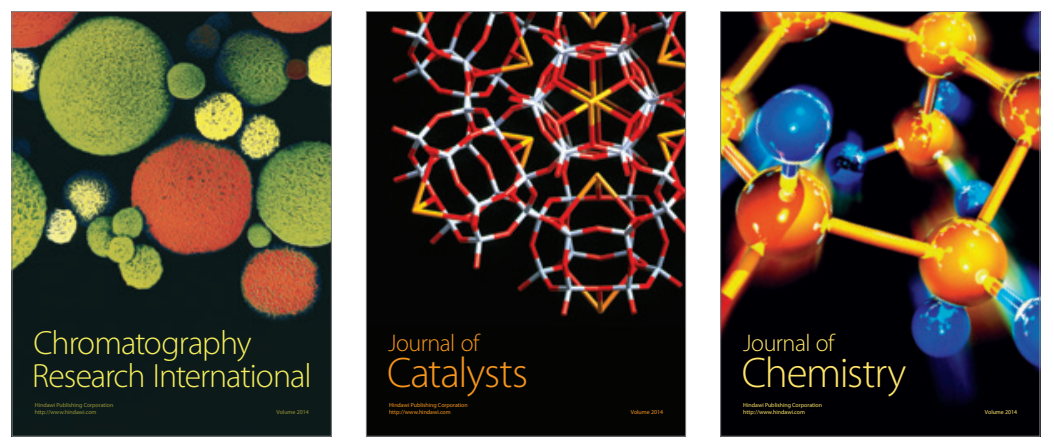
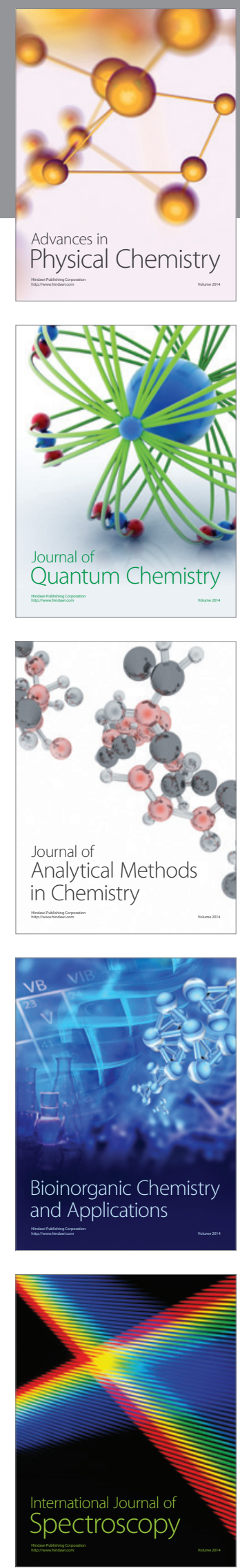